\title{
CONSIDERACIONES ACERCA DE LA DATACIÓN DE UN «MEMBRUM DISIECTUM» PROCEDENTE DEL MONASTERIO DE LAVAIX
}

\author{
Francisco M. Gimeno Blay
}

\section{INTRODUCCIÓN}

La datación de un manuscrito, completo o incompleto, no es, como generalmente se piensa', un problema meramente de erudición, que, por su misma naturaleza afecte exclusivamente a algunos investigadores. Aunque es cierto que los únicos que se ocupan de una forma continua de estos aspectos han sido los paleógrafos, constituyendo para ellos ...un des problémes les plus ardus et au même temps des plus intéressants qui se presentent... ${ }^{2}$, no es menor la importancia que se desprende de la resolución de los problemas planteados por estos restos de la producción literarioideológica de nuestro pasado. A través de la correcta o aproximada datación nos será permitido establecer el stemma codicum, conocer las vías de transmisión de un determinado texto, acercarnos al conocimiento de la producción ideológica de un período determinado, o saber cuáles eran los textos literarios que interesaban a una sociedad concreta y que por ello se transcribian a fin de abastecer el scriptorium a una parte de los Oratores, y más concretamente a la exigua parte que realmente participaba de los dos

\footnotetext{
1 No les son desconocidas a los paĺografos ocupados en esta tarca las críticas procedentes de determinados investigadores que, al no reconocer la necesidad de localizar espacio-temporalmente la producción literaria del pasado, cometen errores de situación y planteamiento.

${ }^{2}$ Cf. MUNDÓ, A.M. «Méthode comparative-statistique pour la datation des manuscritss, Pälaographie, 1981, Colloquium des Comité Intemational de Paléographie, Mínchen, 15-18, September 1981, Herausgegeben von Gabriel Silagi, 1982, bei der Arbeo-Gesellschaft, München, p. 53.
} 
importantes medios de comunicación intelectual del Medievo: la escritura y la lectura ${ }^{3}$.

Ante cualquier membrum disiectum, desprovisto de datación directa, el paleógrafo se cuestionará cuál deberá ser el procedimiento a seguir en la datación. La resolución de este problema es complicada, máxime cuando todavía quedan por delimitar aspectos metodológicos fundamentales que se refieren a la concepción de la Paleografía en general y a la consideración de las escrituras en particular, como son: el ambiente donde surgieron, la sociedad y las personas que las utilizaron, y un cúmulo de cuestiones todavía hoy sin enunciar.

La preocupación por la antigüedad de las escrituras, dirían nuestros eruditos dieciochescos, ha sido una constante que ha afectado a la humanidad entera desde el mismo momento en que el hombre utilizaba la escritura como un elemento de fijación y transmisión de unos pensamientos y/o derechos patrimoniales. No podrá, por lo tanto, sorprendernos que sea precisamente en el siglo XVIII español cuando se inicie la publicación de colecciones documentales e incluso exista un cuerpo de revisores de letra antigua $a^{4}$, cuya misión sea la identificación de un texto escrito de cualquier naturaleza, y la adscripción a un período histórico concreto. No interesaba el procedimiento metodológico seguido sino la fijación de una objetividad, y la consiguiente adscripción de un determinado texto a un tiempo y una sociedad (no olvidemos que, tratándose de documentos, significa la garantía o no de posesión de unos bienes y de los derechos que de ella se desprendían). Junto a la peritación paleográfica nació precísamente la Diplomática como crítica de verdad o falsedad. Fruto de esta preocupación, el P. Merino de Jesucristo se preocupó, ya en el siglo XVIII, por disponer de forma cronológica los facsímiles en su manual porque esto servía:

... no poco para confundir las escrituras legítimas y genuinas, con las que no lo son, y que pudo falsificar la malicia y asi disponemos la obra por orden cronológico ... ${ }^{5}$

${ }^{3}$ De este aspecto ya nos hemos ocupado en otro trabajo aLa escritura en la Cartuja. Análisis diacrónico a través de las Constituciones», SAITABI, XXXIII (1983), p. 5-17. El estudio de los textos legislativos cartujanos permite observar los diferentes grados de conocimiento de la escritura entre los monjes profesos de esta religión, y cómo la oposición real conocimientodesconocimiento revela una diferenciación social, considerando a las personas en tres fases de su vida: nacimiento, ingreso en la Orden, y trabajo a desempeñar dentro de la Religión.

${ }^{4}$ Cf. COTARELo Y MORI, Emilio, Diccionario biográfico y bibliográfico de caligrafos españoles, tomo II, Madrid, 1916, artículo 918, p. 196-198.

${ }^{5} \mathrm{Cf}$. M desde la entrada de los Godos en España hasta nuestros tiempos, Madrid 1780, fol. $2 \mathrm{r}$. 
Si bien la necesidad de datación y localización de manuscritos se había sentido desde antiguo, habría que esperar hasta el presente siglo para que naciera el Comité Internacional de Paleografía, y se propusiera, en su sede, por primera vez, la publicación completa y sistemática de todos los manuscritos datados con certeza o aproximadamente ${ }^{6}$. La empresa de recogida de material para la conformación del gran archivo paleográfico que sirviera de base para efectuar futuros estudios sobre dataciones, difusión del tipo escriturario e interconexiones culturales entre las distintas provincias escriturarias, tuvo bien pronto una fuerte acogida en ciertos países europeos en los que se está llevando a cabo, o ya se ha realizado, la publicación de esos importantes repertorios fotográficos ${ }^{7}$. A pesar de la edición de esos catálogos, de los cuales España no se ha ocupado de forma definitiva, el problema de las dataciones de manuscritos carentes de fecha directa sigue preocupando a los paleógrafos quizá más que antes de la celebración del primer Congreso. Fruto de esa inquietud se han publicado ciertos trabajos, en cierto modo con miras metodológicas, para afrontar esta delicada cuestión. Entre ellos cabe destacar los recientes de Mundó, Gilissen, y otros sobre escrituras determinadas, como el de Petrucci sobre la Uncial, y de Autenrieth sobre la Carolina.

El objeto de la publicación de estos repertorios de reproducciones de manuscritos tenía y tiene, todavía hoy, un evidente interés. Las muestras publicadas con indicación de fechas, directa o indirectamente, permitirán y constituirán la base de comparaciones futuras y al mismo tiempo serán el instrumento necesario para datar los que se presentan carentes de indicaciones e indicios que permitan adscribirlos a un espacio-tiempo. A pesar de

${ }^{6}$ Las propuestas del primer congreso quedaban expuestas en L'Avant-Propos, entre otros aspectos no menos interesantes para el futuro de la Paleografia, del siguiente modo: «...l'établissement d'un project de catalogue photographique des manuscrits datés, écrits en écriture latine, des origines au Xvi sic̀cles.

Cf. Nomenclature des écrimures livresques du $x x^{f}$ au Xvf siecle. Premier Colloque international de Paléographie latine, Paris, 28-30, Avril, 1953, Publications de la CNRS, París. 1954, p. 3. El proyecto quedaba más explicitado en el punto IV titulado Catalogue des manuscrits datés, id., p. 5 .

7 Una detallada exposición de los países que han acogido el proyecto e iniciado la publicación puede consultarse en M.C.GARAND, Le Catalogue des manuscrits datés: bilan et perspectives, Pallaographic, 1981, p. 25-29.

${ }^{8}$ Cf. Mundó, A.M. «Méthode comparative-statistique...»; Gin. ISSEn L. «Analyse des écritures: manuscrits datés et expertisse des manuscrits non datésw, en Les techniques de laboratoire dans l'étude des manuserits, París, 13-15, septembre 1972, Editions de la CNRS, París 1974. p. 25-35. Discusión 35-40; PETRUccl, A., «L'Onciale Romana. Origini, sviluppo e diffusione di una stilizzazione grafica altomedievale (sec. VI-XI)* STUDI MEDIEVALI. XII (1971), 75-135; AUTENRIETH, J. *Probleme der Lokalisierung und Datierung von splitkarolingischen Schriftens, CODICOLOGICA, 4 (Leiden, 1978), p. 67-74. Recientemente se ha ocupado del tema A. PetrucC, La descrizione del manoscritto, Storia, problemi, modelli, Roma 1984, p. 49-54. 
que dispongamos de estos catálogos, el paleógrafo se ve obligado a recurrir ineludiblemente a los métodos tradicionales, es decir, a la inspección ocular, excepto en los casos propuestos por Gilissen y Mundó, y tomando siempre como base el saber o la pericia adquirida a lo largo de los años de dedicación al tema. Se trata, por tanto, de establecer una comparación entre el manuscrito por datar y los publicados que presentan datación directa.

Este complicado problema se agudiza todavía más cuando nos referimos a manuscritos españoles, e incluso más al cerrar el círculo intentando estudiar la provincia escrituraria o KOINÉ gráfica representada por la Corona de Aragón durante la Edad Media, en primer lugar por las diferencias existentes con los provenientes de otras provincias escriturarias de la misma época, y en segundo lugar porque los grandes manuales de Paleografía Española prestaban escasa atención a los centros escriturarios de la Corona de Aragón, hecho motivado posiblemente por una despreocupación de determinados temas en relación a la geografía y a la concepción de la ciencia de estudio".

Así, pues, a la datación de los textos escritos en la Corona le afectan dos problemas, uno general y otro particular: el primero se desprende de la esencia misma del hecho de la datación. La cronología de la transcripción, copia y/o confección de un determinado texto es como tal una realidad evidente; podríamos decir, incluso, que se trata de una objetividad, un hecho histórico concreto y determinado. Frente a ella el paleógrafo, al intentar adscribirlo a un espacio-tiempo, ofrece y utiliza como único medio de trabajo una subjetividad perceptible porque su modo de proceder es empírico e hipotético, difícilmente exacto, y en modo alguno cuantificable $^{t}$. En este estudio se produce, por consiguiente, una desconexión y al mismo tiempo un enfrentamiento entre objetividad, fecha de copia-redacción de un texto, y la subjetividad, representada por las categorías de conocimiento creadas por el hombre para poder aprehender mejor lo cognoscible.

El segundo problema, particular, en la medida en que el texto a datar se localiza dentro de la KOINÉ escrituraria de la Corona de Aragón, reside y se origina en la carencia, casi absoluta, de infraestructura y de base material de textos publicados que permitan establecer las comparaciones necesarias

9 Así se puede observar en los manuales de MILLARES, FLORIANo y el de la UNED. Para todos ellos la Paleografía no dejaba de ser más que una disciplina universitaria con un marcado acento auxiliarista.

${ }^{10}$ No obstante, existen intentos de hacer matematizable la escritura; aunque no sabemos si con un determinado interés se pretende olvidar el dinamismo inherente al fenómeno escriturario en su conjunto. La ciencia de las escrituras dificilmente se conducirá dentro de unos límites estrictamente matemáticos. Será utilizado posiblemente para la identificación y discernimiento de manos que saben escribir, como demuestra L. Gll ISSEN en L'Expertise des écrimures médiévales, y que interpretan una escritura determinada, un cóđigo gráfico, pero no podrá ser considerado como el final de la investigación paleográfica. 
en toda datación de manuscritos carentes de fechas, y de los más complicados "membra disiecta", que todavía hoy pueden encontrarse en archivos, bibliotecas, e incluso colecciones privadas. Respecto a la publicación de archivos paleográficos que reproduzcan facsímiles referentes a la Corona de Aragón, sólo disponemos de los trabajos de conjunto de García Villada, Millares Carlo, Laminas de la Cátedra de Madrid, Canellas, y solamente de uno de carácter particular y exclusivo: la Colectánea de las profesoras Mateu Ibars"

No obstante, existen otras publicaciones paralelas, que no siendo de carácter esencialmente paleográfico, adjuntan reproducciones documentales que pueden servir de comparación.

El panorama, si se considera en su justa medida, es bastante desolador; pero, ¿a qué es debida la carencia de este utillaje tan elemental? No es erróneo pensar que la causa de esta falta de materiales sea debida al carácter eminentemente práctico y auxiliarista que se le ha atribuido, desde antiguo, a esta disciplina universitaria, y que sólo debía cumplir dos requisitos fundamentales:

- enseñar a leer-descifrar escrituras antiguas a los estudiantes universitarios en la medida que futuros investigadores, $y$

- preparar, mediante la transcripción, la edición de los textos necesarios a los investigadores como base de sus estudios.

Injustificadamente y desde estos presupuestos se ha querido, y todavía hoy se quiere, inferir la necesidad de su estudio. No habrá, sin embargo, que estancarse en considerar únicamente la necesidad de estudio, puesto que ello conlleva la reducción de las posibilidades de trabajo e investigación.

Vistos los problemas y objeciones realizables al único método de datación de que se dispone actualmente ${ }^{12}$, se pretende solucionar el del manuscrito que hoy nos ocupa de la siguiente manera: primero, deberemos situar

11 Garcia Vulada, Z., Paleografía Española. Precedida de una introducción sobre Paleografta latina, Reed. Barcelona 1974; Mil.ares CARLo, A., Tratado de Paleografía española, Madrid 1932: CANELLAS LOPEZ, A., Exempla Scripturarum latinarum. Pars altera, Caesaraugustae 1974: MATEU IBARs, J. y M.D., Colectánea paleográfica de la Corona de Aragón, siglos DC:xVm, Barcelona Universidad, 1980.

12 A pesar de todas sus limitaciones también utilizamos el sistema comparativo, e ínciuso lo desarrollamos aquí, en la datación de otro manuscrito. En el trabajo titulado Notas acerca de la datación del manuscrito Constituciones sinodales de los obispos Fray Sancho d'UIl. don Elias y don Juan de Barcelona, publicado en los Estudios dedicados al Dr. Juan Peser AlfixAnDRe, Universidad de Valencia 1982, vol.II, p. 167-176. En él y en una de sus conclusiones ya se planteaba que tanto las estimaciones numéricas como las oculares se debían considerar como meras aproximaciones a una realidad y no como objetivaciones de la misma. 
el texto en un período escriturario determinado, a pesar de no olvidar que las Nomenclaturas paleográficas son meras categorías de conocimiento, y que acaban limitando todo el dinamismo inherente al fenómeno escriturario. Después, y tras efectuar un análisis comparativo de todos los elementos que lo integran, intentaremos acercarnos en el más alto grado posible, al tiempo de confección del códice al que pertaneció este membrum disiectum.

\section{DATACIÓN}

\section{EL TIPO ESCRITURARIO}

El primer elemento a reconocer en todo intento de datación debe ser el tipo escriturario en el que se sitúa el manuscrito, la adscripción, en definitiva, a un período general de la evolución de la escritura latina ${ }^{13}$. Esto nos proporciona unos límites globales de análisis, términos a quo usque ad, en los que se incluiría la redacción o copia del texto.

Definamos, pues, la caligrafía utilizada. El texto presenta una escritura gótica, cuyos caracteres se observan principalmente en la descomposición de curvas, reduciéndolas a ángulos (caso de las letras que llevan aquéllas). Sirvan de ejemplo las que aparecen en la $a, c, d, e, o$, y especialmente en la descomposición de los trazos de algunas mayúsculas: $A, D, Q, U^{14}$.

Situado el manuscrito dentro de la escritura gótica, es decir, entre finales del siglo XI y principios del siglo XV. debera intentarse una delimitación más precisa. Este encuadramiento supone la aceptación de categorías

13 Para el conocimiento de estos períodos evolutivos de la escritura el investigador ha utilizado siempre una Nomenclatura. A pesar de que ellas han constituido una preocupación fundamental de los paleógrafos no debemos olvidar que se trata de una adaequatio rerum ad mentem, un intento, en definitiva, de disciplinar y regular lo cognoscible bajo unos esquemas mentales.

14 *La plume biseautée à gauche, permettant au scribe de prendre sans cesse appui sur toute la largueur du biseau, sauf pour les traits horizontaux ou franchement obliques, donne una alternance régulière de pleins et de déliés; si le mouvement des courbes est très ample, il se traduit par une angulosité marquée, qui se produit au moment où le trait change de sens - le fracture - et....; vid. BOLSSARD, J., Influences insulaires dans la formation de l'écriture gothique, SCRIPTORIUM, V (1951), p. 243. 
nomenclaturísticas más complejas ${ }^{15}$, lo cual permitirá reducir el espacio de probabilidades para la datación, y acercar, en definitiva, los términos a quo - usque ad.

Veamos cuáles han sido los calificativos que le han añadido los paleógrafos a este tipo de escritura. Kirchner la incluiría dentro de las que él denomina litterae textuales, y que, según su colección de láminas, se sitúa entre 1151 (Tabula, 1) y 1472 (Tabula, 36).

Según las nomenclaturas utilizadas por A. Canellas, este fragmento se podría localizar entre las pregóticas como base de las que posteriormente darían lugar a las textuales caligráficas. Las pregóticas están recogidas en su colección por los facsímiles XXXVII del siglo XII, y XXXIX, de 1171, del área oriental peninsular. En lo que respecta a la textual caligráfica redonda, se puede localizar entre 1218 y 1510 (láms. XIV, XLVI, XLVII, XLVIII, XLIX, L y LI). Incluye otra - núm. LII-con la única marca de datación de saec. XVI. Esta última, y alguna de las anteriores caen, evidentemente, fuera del ciclo escriturario gótico, y pueden considerarse como viejas reminiscencias de tiempos pasados. Podría pensarse, incluso, que se le está confiriendo de alguna manera otro valor a la escritura que el puramente transmisor de un pensamiento, posiblemente el estético y de consideración.

Atendiendo a la periodización que presentan las profesoras Mateu Ibars en su Colectánea, el manuscrito objeto de estudio se situaría entre dos de los ciclos enunciados alli, el cisterciense: ciclo monacal (s. XII) y el gótico: ciclo gótico mediterráneo (s. XII). Es en el tránsito de estos dos períodos donde se sitúa el texto que pretendemos datar, como más adelante se expondrá.

La larga duración de las góticas textuales, atendiéndonos a las colecciones de facsímiles publicados por I. Kirchner y A. Canellas, no nos permiten

15 El tema de las nomenclaturas de la escritura gótica fue tratado no muy satisfactoriamente por G.I. LIEFTINCK en el primer congreso internacional de Paleografía («Nomenclature des écritures livresques du $\mathrm{x}^{\mathrm{e}}$ au $\mathrm{xvr}^{\mathrm{e}}$ siecles, Premier Colloque International de Paléographie Latine, París, 28-30 avril 1953, Paris 1954. Su comunicación lleva por título: Pour une nomenclature de l'écriture livresque de la période dite Gothique. Essai s'appliquant spécialement aux manuscrits des Pays-Bas médiévaux, p.15-34. Una crítica de las tesis defendidas por el mencionado autor puede consultarse en DESPY, G., «De la terminologie palóographique médiévale. A propos d'un ouvrage recentes, Revue Belge de Philologie et d'Histoire, XXIV (1956), p. 174-181; y un comentario no demasiado correcto realizado por PAVEL SPUNAR «Sur les questions de la terminologie paléographique des écritures livresques du $9^{e}$ au 16 siècles, Eunomia, 1 (1957) 35-40, 95-97. El tema también ha sido tratado por J. KIRCHNER en Scriptura Gothica Libraria. A saeculo XII usque ad finem medii aevi, LXXXVII imaginibus illustrata. Monachi et Vindobonae, MDCCCCI.XVI. Sin embargo, los que más nos interesan son los de CANELLAS LOPE, A., Exempla scripturarum latinarum in usum scholarum, Pars altera, Caesaraugustae, MDCCCCL XXIII, y el de las profesoras MATEU lBARS, J y M.D., Colectánea paleográfica de la Corona de Aragón. Siglos IX-XVIII, Vol. II, láminas, Barcelona Universidad, 1980. 
una mayor aproximación, aunque nos sirven para circunscribirnos a un solo tipo escriturario, y por consiguiente, a localizar, en los manuscritos que utilizaran este tipo, determinados elementos gráficos que nos permitieran acortar o delimitar todavía más el período cronológico en que se escribió el membrum disiectum procedente de Lavaix, al menos en lo que se refiere a su conservación archivística.

Un elemento gráfico nuevo nos permitiría reducir el espacio cronológico de copia del presente manuscrito. Se trataba de la utilización de la s sobrescrita sin valor abreviativo. Ello nos situaba en el siglo XII si aceptábamos la datación que este fenómeno le daba A. Cappelli, quien se expresaba en los siguientes términos: «La s trovasi spesso sovrapposta in fine di parola, specialmente nel XII secolo, per rappresentare puramente sé stessa...." ${ }^{16}$

El mismo fenómeno observábamos en nuestro manuscrito en las siguientes palabras:

$$
\begin{aligned}
& \text { nos (col. 1, 1. } 18 \text { y 21); } \\
& \text { filios (col. 1, 1.18 y 21); } \\
& \text { vos (col. 2, 1.26); }
\end{aligned}
$$

iniquitatos (col. 2, 1. 3);

magis (col. 2, I. 26);

donde las $s$ finales se encuentran sobrescritas y sin ningún valor abreviativo, fenómeno que también hemos localizado en otros manuscritos del siglo XII, donde aparecía:

[a] En 1151, en la palabra Necones, de la lista de Seniores et tenentes, en un documento de Ramón Berenguer IV, datado en Zaragoza y escrito por Pedro de Capella ${ }^{17}$.

[b] En 1164, en el verbo loqueris (1. 3), utilizado en el documento de la consagración de la iglesia de Sant Romà de les Bons y expedido en la parroquia d'Encamp, en Andorra ${ }^{18}$.

[c] En 1166, en la desinencia del genitivo del nombre Uguonis en una carta compositionis entre el abad del monasterio de Montearagón y Bernardo abbate sancte Marie de Oliva ${ }^{19}$.

$16 \mathrm{Cf}$. CAPFE, Lexicon Abbreviaturaram. Dizionario di abbreviature latine ed italiane, Milano, $6^{2}$ ed., 1973, p. XIIV

17 Cf. Canel. Álbum facsímil núm. 3. A partir de este documento estamos realizando un trabajo sobre la introducción y difusión de la escritura gótica en la escribanía de Ramón Berenguer IV. El trabajo lleva por título: ¿ Formas gráficas góticas en el siglo xu? A propósito de un documento de Ramón Berenguer IV, (en prensa).

IB Cf. Mateu IBars, J. y M.D., Colectánea paleográfica de la Corona de Aragón. Barcelona 1980, 1ám. 58. El pergamino reproducido se conserva en el Archivo Capitular de Urgel.

${ }^{19}$ Cf. Mateu Ibars, J. y M.D., op. cit., lám. 60. El documento original en Archive Histórico Nacional (AHN), Monasterio Jesús Nazareno de Montearagón (Huesca), carp. 624. núm. 14. 
[d] En 1169 y en pergamino del Archivo del Pilar de Zaragoza, encontramos la $s$ volada sin valor abreviativo en las siguiente voces:
- $\operatorname{tres}(1.8)$
- $\operatorname{suos}(1.13)$
- pezas (1.8)
- partes (1.14)
- andachales (1. 15)
- $\operatorname{parts}(1.11)$
- Arolas $(1.15)^{20}$

[e] En 1176 y en un pergamino conservado en el mismo archivo observamos que casi todas las palabras llevan esta letra como final. Así aparece:

$\begin{array}{lll}\text { - donacionis (I.1) } & \text { - facitis (fd.) } & \text { - possideatis (id.) } \\ \text { - vos (1.2) } & \text { - afrontaciones (1.4) } & \text { - totos (1.7) } \\ \text { - facistis (I.3) } & \text { - exiis (1.5) } & \text { - homines (id.) } \\ \text { - iacensis (id.) } & \text { - regresiis (id.) } & \text { - auditores (id.) } \\ \text { - dedistis (id.) } & \text { - suis (id.) } & \\ \text { - alios (id.) } & \text { - abeatis (id.) }\end{array}$

[f] También el Liber Feudorum Maior (1192) del reinado de Alfonso II es ilustrativo para datar este fenómeno gráfico. Tomando como muestra las láminas publicadas por Mundó, encontramos la $s$ sin valor abreviativo en las siguientes palabras:

- suos (lám. 8, col. 1, 1.8)

- Ermengaudis (lám. 12, col. 2, 1.12)

- elemosinarios (lám. 10, col. 2, 1. 5) - Rossilionensis (lám.14, col, 1, 1. 15)

- his (lám. 11, col. 2, 1. 1)

- nos (lám. 14, col. $1,1.19)^{22}$

[g] La lámina correspondiente al fol, 51 r, del manuscrito M.III.2. de la Biblioteca de El Escorial, del año 1188, publicada por I. Kirchner, en donde también aparecen las $s$ voladas sin valor abreviativo:

- ingenuis (1. 10)

- alegis (1.23)

- $\cos (1.12)$

- copulandas (1.25)

- heredes $(1.18)^{23}$

${ }^{20} \mathrm{Cf}$. MATEu IBARS, J. y M.D., op. cit, lám. 61.

${ }^{21}$ Cf. MATEu IBARS, J. y M.D., op. cit., lám. 63(a).

22 Cf. Mundo, A.M., «El pacte de Cazola de 1179 i el "Liber Feudorum Maior". Notes paleogràfiques i diplomàtiques», publicado en «Jaime I y su época, 1 y 2n, Zaragoza 1980 , p. 119-129.

${ }^{23}$ Cf. KIrchner, I., Scriptura Gothica libraria. A saeculo XII usque ad finem medii aevi. LXXXVII imaginibus illustrata. Monachii et Vindobonae, MDCCCCLXVI, lám. 6; vid. también p. 20-21. Cf. Antot., G., Catálogo de los códices latinos de la Real Biblioteca del Escorial. 
[h] Finalmente también la hemos localizado en el fol. $44 \mathrm{v}$.. del manuscrito 145 de la BP de Tarragona, que contiene las Collationes Patrum de Johannes Cassianus. Aparece en la palabra aliis (col. $2,1.22)^{24}$.

La coincidencia de la datación de esta $s$ entre Cappelli y la supuesta provincia escrituraria de la Corona de Aragón nos delimita el campo de acción en el que nos teníamos que mover para efectuar las comparaciones necesarias.

De una forma tan simple, es decir, por el hecho de haber utilizado esta s sin valor abreviativo nos habíamos delimitado el campo de acción al siglo XII. Faltaba por consiguiente que analizáramos durante este período todos los facsímiles reproducidos para el área escrituraria a la que presumiblemente pertenecía el membrum disiectum. Sabíamos de antemano que el lugar de conservación archivística había sido el monasterio de Lavaix, en el Pirineo, y más concretamente en la comarca del Pallars, según nos advirtió J. Trenchs ${ }^{25}$. Por ello debíamos limitarnos a los manuscritos procedentes del área oriental peninsular y escritos en el siglo XII. No resultaba, por consiguiente, difícil establecer la comparación y la selección del material utilizable.

Analizaremos a continuación todos aquellos elementos del alfabeto, abreviaturas, nexos y ligaduras que nos hayan servido para datar el manuscrito.

\section{EL ALFABETO}

\section{Mayúsculas}

La utilización de las mayúsculas es muy limitada. Son pocos los ejemplos que aparecen, y en su mayoría son tipos minúsculos agrandados para conseguir el efecto óptico de la mayúscula. Minúsculas agrandadas son la $\mathbf{E}$ (col. 1, 1. 27); la $\mathrm{H}$ (Hic, parte carnis, col. 2, 1. 25), diferenciándose del resto de $h$ porque presenta al pie dos refuerzos de dirección izquierdaderecha. Otro tanto sucede con la $\mathrm{M}$ de la palabra tuam (parte pili, col. 2, 1. 12), donde esta letra, aun siendo minúscula, recibe un tratamiento especial en su último tramo, que la hace similar, al menos en la primera parte, al trazo final de la $M$ uncial, finalizando con una prolongación desmesurada, uniéndose a los signos de puntuación (dos puntos y una

24 Cf. Mateu IBARs, J. y M.D., op. cit., lám. 73.

${ }^{25}$ Este profesor supo que la procedencia era Lavaix porque en el lugar donde él lo compró vendian asimismo otros documentos que afirmaban este origen. 
coma). Lo mismo podríamos decir de la $N$ agrandada con valor de mayúscula (Non, p., c., col. 1, I. 14).

Otras, por el contrario, como $A, F, P, R, S, T, V$, y especialmente $D, O$ y $Q$, han supuesto una investigación mayor a fin de localizar en ellas posibles elementos de datación. Los resultados han sido los que exponemos a continuación:

[a] La $a$ uncial se presenta de una forma muy afectada por el tratamiento gótico. El contraste de trazos es evidente. Se aprecia en las palabras siguientes: adhuc (p.c., col. 1, 1. 13), Alioquin (p.c., col. 2, 1. 18 y p.p., col. $1,1.8$ y 25) y tuam (p.p., col. 21.12 ).

Tratamientos similares los encontramos también en:

[a.1] En 1151, en la voz Arral, en el documento ya citado de Ramón Berenguer IV. Esta palabra aparece en la lista de seniores et tenentes del escatocolo ${ }^{26}$.

[a.2] En 1164, en la palabra Anno (I. 16), en el documento ya comentado de zona andorrana $\mathrm{a}^{27}$.

[b] Merece especial atención la forma adoptada por la $F$ en la palabra

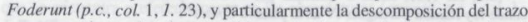
curvo superior en ángulos a medida que la pluma va deslizándose sobre el pergamino.

[b.1] En 1151, en la palabra Facta del escatocolo ${ }^{28}$.

[b.2] En 1164 y 1166 , en dos chirógrafos o cartas partidas, especialmente la parte superior de la letra ${ }^{29}$.

[b.3] En 1169, en la palabra Fortion ${ }^{30}$.

[b.4] En un códice datado en el siglo XII en el subjuntivo de Facio $\left(\right.$ fit, 1. 26) ${ }^{31}$.

[c] Los tratamientos que presentan las mayúsculas $P, R, S, T$ y $V$ también están afectados por las formas góticas y son perfectamente localizables en manuscritos coetáneos:

[c.1] La $P$ mayúscula que aparece en post (p.p., col. 1, 1. 14) es bastante similar a la utilizada en la palabra Petro del documento de 1151 de Ramón Berenguer IV ${ }^{32}$.

${ }^{26}$ Cf, Canellas, A. (ed.) Colección Diplomática, lám. 3.

${ }^{7}$ Cf, MATEU IBars, J. y M.D., Colectánea, lámina 58.

${ }^{28}$ Cf. Canel.las, A., (ed.), op, cit., lám. 3.

${ }^{29}$ Cf. MATEL IBARS, J. y M.D. , op. cit. láminas 59 y 60.

30 Cf. Mateu IBARs, J. y M.D. . op. cit., lám. 61 , línca 4.

31 Cf. Mateu IBars, J. y M.D., op cit, lám 74. Reproduce el manuscrito 20 , fol. 20 v. del A.C. TORTOSA.

32 Cf. CANellas, A. op. cit., lám. 3, y especialmente la suscripción del escribano Petro Capella. Este amanuense también la utiliza en el nombre Petro Castellaçuelo, del escatocolo y en otras $P$ a lo largo del documento. 
[c.2] La $R$. de procedencia capital utilizada en la palabra regem (p.c., col. 1, 1. 6-7) también presenta el mismo tratamiento que la utilizada por Petro Capella en el nombre Raimundus ( 1.1 ) del documento de $1151^{33}$.

[c.3] Igual sucede con la $S$ mayúscula de nuestro membrum desiectum de la abreviatura sed (col. 1, 1. 2) y con la empleada por Pedro Capella en el nombre Sancio del escatocolo ${ }^{34}$.

[c.4] También las formas $T$ (Testamentum, col. 2, 1. 16) y de la $V$ con un primer trazo excesivamente prolongado sobre la línea de escritura (Unde, p.c., col. 1, 1.5), son localizables en los manuscritos coetáneos ${ }^{35}$.

[d] Mención especial merecen las soluciones adoptadas por las letras $D$ (Dixerunt, p.c., col. 1, 1. 7; Dominum, p.c., col. 1, 25), $O$ (offerebat, p.c., col. 1, I. 9-10), y Q (Quanta, col. 1, I. 1, que y quid, col. 1, 1. 2; Quomodo, col. 1. 1. 20 y también en 21), donde las curvas existentes en la formación de las letras se ven afectadas por la rotura de éstas y su descomposición en trazos más cortos donde se aprecian claramente los puntos de ataque y el final de todos los trazos. Un fenómeno semejante se puede observar:

[d.1] En 1151, en la $O$ utilizada por Pedro Capella en la suscripción del conde-rey Ramón Berenguer IV en la palabra Comes $^{36}$.

[d.2] En 1188, en la $Q$ de quodcumque del manuscrito de los Fori iudicium ${ }^{37}$.

\section{Minúsculas}

En lo que respecta a las formas adoptadas por el alfabeto minúsculo, también hemos realizado una búsqueda de los elementos más significativos, letras más características, que a su vez nos permitían acercarnos a la datación del manuscrito. De ahí que no consideraremos todas las formas adoptadas por las letras, sino únicamente aquellos elementos que las individualizan, haciéndolas peculiares.

[1] Debemos considerar, en primer lugar, la forma que presentan todos los alzados en sus respectivos puntos de ataque o de apoyo de la pluma

${ }^{33}$ Cf. CANetlas, A., op. cit., lám. 3, línea 1.

${ }^{34} \mathrm{Cf}$. id., línea 4 de la lista de Seniores.

${ }^{35} \mathrm{La} T$ la hemos localizado en un manuscrito de 1171 publicado por A. CANELLAS, Exempla Scripturarum latinarum, Pars altera, Zaragoza 1974, facsímil número XXXIX, columna 1. línea 10 (tanto). La forma adoptada por la $V$ también se ha localizado en 1164 en la palabra Vox (línea 8), Cf. MATEu IBARs, J. y M.D., Colectánea, lám. 58, y también en la 37 (Vnde, línea 17) del siglo xII, y en la 39 de 1171 (Videmus, columna 2, línea 21) de los Exempla de A. Caneulas.

${ }_{36}$ Cr. Canellas, A. (ed,), Colección diplomática, lám. 3.

37 Cf. KIRchner, I, Scriptura gothica libraria, lám. 6, línea 4. 
sobre el soporte. Nos muestran, la mayoría, un trazo inclinado de dirección derecha-izquierda al comenzar el descenso en forma de triángulo. Se pueden apreciar perfectamente en las letras: $b, d, l, h$, en el caído de la $p$, y en el primer trazo de alguna letras que no sobresalen de la caja de escritura. El mismo tratamiento reciben los alzados de algunos de los facsímiles consultados de los años $1151^{38}, 1164,1166,1169,1173,1176,1179,1181,1193^{39}$.

Del mismo modo debían considerarse también otras letras que presentan algunos elementos muy particulares y que son fácilmente localizables en otros manuscritos coetáneos. La comparación de todas estas características nos ha dado el siguiente resultado:

[2] La $A$ aparece realizada de dos formas, principalmente: la carolina con un tratamiento gótico que nos presenta un primer trazo descrito sobre la línea de escritura antes de realizar el descenso y que sirve de elemento de unión con la precedente (quanta, p.c., col. 1, 1. 1); cuando esta letra no va unida a la anterior, este trazo resulta innecesario y por consiguiente no aparece (frenaretur, p.c., col. 1, l. 1); y un segundo tipo, el de la cursiva, que se encuentra preferentemente en las abreviaturas (quanta, p.c., col. 1, 1. 1; $V T$ en p.c., col. 1, 1. 4).

Las formas, al menos las empleadas por el primer tipo, son localizables perfectamente en el siglo XII y en todos los manuscritos con los que hemos comparado elementos anteriores, que venimos tomando en consideración ${ }^{40}$.

[3] Las letras $B$ y $C$ también se han visto afectadas por el mismo tratamiento gótico que ha influido a todo el alfabeto. Solamente merece destacarse la descomposición del trazado que sufre el primer tramo de la $b$, apreciándose especialmente en sibi (p.c., col. 1, 1. 20) y ubi (p.c. col. 2 , 1. 14),

Respecto a la $c$ y a su peculiar forma adoptada en las ligaduras, se tratará en el apartado dedicado a su estudio.

[4] La $D$, como es normal, presenta dos formas, una procedente de la uncial, pero influida profundamente por el tratamiento gótico (quid, p.c., col. 1, 1.2), y otra minúscula (dixerunt, p.c., col. 1, 1.2). Interesa destacar en esta última la presencia de dos triángulos en la parte izquierda del alzado: el superior como punto de ataque del primer trazo; $y$ el otro a la

38 Cf. CANFllas, A. (ed.), Colección diplomática, lăm. 3.

${ }^{39}$ Los años incluidos entre 1164 y 1193 se corresponden con las siguientes láminas publicadas por MATEU IBARS, J. y M.D. en la Colectánea: núm. 58, 59, 60, 61, 62, 63 a y b, 64, 67,73 y 74 .

40 Cf. Caneu is, Colección Diplomática, facs. III, MAteu IBARS, J. y M.D., Colectánea, lán. 67, 73. 
mitad de la altura, que respondería también a la misma función, peroen este caso para la realización de la curva de la izquierda (dixerunt, p.c., col. 1,1 . 2; dignum, p.c., col. 1, 1. 11, y otras) forma que también hemos localizado en otros manuscritos de la misma época, es decir, durante el siglo XII, perviviendo incluso en algunos manuscritos del siglo XIII, como lo demuestra el de 1240 , reproducido por A. Canellas ${ }^{41}$.

[5] De la $G$, considerada como una letra significativa, poco podríamos decir, sólo que ha sido formada por una $O$ donde las curvas se han descompuesto en dos trazos, cóncavo y convexo, y que a esta parte se ha unido el caído.

[6] Tiene interés, por el contrario, la $I$, y más concretamente, los puntos diacríticos que la acompañan: misterii (p.c., col. 2, 1. 27), Alioquin (p.p., col. $1,1.8$ y 25) y Hostiis (p.p., col. 1, l. 22).

La importancia de la presencia de estos signos radica en el hecho de que Battelli localizaba esta característica gráfica solo a partir del siglo XIII.

Afirmaba: «La $i$ nel sec. XIII ha sopra il trattino diacritico anche quando é isolata; nel XIV comincia ad apparire il puntino in luogo della lineettas ${ }^{42}$.

La comparación con otros manuscritos del siglo XII nos ha permitido observar que el uso de la línea diacrítica aparecía ya en el período anterior al que había señalado el mencionado profesor ${ }^{43}$.

[7] Respecto a las letras $L, M, N, O$, poco podríamos decir. Sus respectivas particularidades quedan inmersas en el conjunto gráfico que afecta a todas las letras ya comentadas.

[8] Sólo queremos destacar en la $Q$ minúscula la presencia de un adomo que nace al final del trazo que describe la cabecera con dirección derechaizquierda (queque, p.c., col. $2, I$. 24), y que también hemos localizado en otros manuscritos del siglo xII. Entre ellos destacaremos:

[8.1] En un manuscrito del siglo XII $(1.11)^{44}$.

${ }^{41}$ Cf. Canellas, A., Exempia Scripturarum, Pars Altera, lám. 46, y especialmente en las palabras: dare, dictum (línea 3).

42 Cf. Battel.LI, G., Lezioni de Paleografia, Città del Vaticano, 3 ed, 1949, p. 226.

43 Los encontrábamos en 1164. Cf. MATEU lBARs, Coleciánea, lám. 58, rogerii (línea 15) febroarii (línea 18) en un manuscrito carolino reproducido en la misma Colectánea (lám. 72. ieiunii (línea 4). También aparecía en algunos faesímiles procedentes del Liber Feudoram Maior, cf. Mundó, El pacte de Cazola, lám. 10, obiï (columna 2, línca 8).

44 Cf. Mateu Irars, Colectánea, lám. 57. 
[8.2] En 1164, en la palabra loqueris (1. 3) de un documento del archivo Capitular de Urgel ${ }^{45}$.

[8.3] En un manuscrito datado entre los siglos XI-XII $(1.19)^{46}$.

[8.4] En 1171, en el relativo que (col. 1, 1. 12) entre otras ${ }^{4}$.

[8.5] En el Liber Feudonum Maior, en la palabra querimonias (col. 2, 1. 19) ${ }^{48}$.

[9] La $R$, como es costumbre en este período, se nos presenta de dos formas, una con ástil vertical al que se une posteriormente la cabecera (martillete); la otra, por el contrario, es la redonda o tironiana que suele aparecer después de una letra curva, pero no necesariamente ${ }^{49}$.

[10] La $S$, como ya es común, presenta dos formas distintas además de la sobrescrita $\sin$ valor abreviativo, y que hemos comentado anteriormente. Frente a la $S$ alta de uso casi exclusivo, aparece la de doble curva, preferentemente al final de palabra, aunque a veces también la encontramos en la composición de alguna determinada voz, pero siempre que la $S$ quede al final de línea.

[11] $T, V, X$, e $Y$ griega no presentan características particulares que nos hayan servido de base para la datación.

\section{NEXOS}

Uno de los elementos a considerar en el análisis de cualquier escritura son los nexos, entendidos como: ... fusioni di segni alfabetici non spontanee in quanto dettate da affinita grafiche, ma pensate e volute prima della scrittura, nelle quali uno o alcuni tratti di una lettera servano anche all'altra... ${ }^{\text {so }}$.

Ateniéndonos a la presente definición de G. Cencetti, dentro de la escritura gótica sólo se pueden considerar como nexos las fusiones de curvas contrapuestas, estudiadas el pasado siglo por Wilhelm Meyer ${ }^{51}$, de

45 Íd. lám. 58.

46 Id., lám. 72.

${ }^{47}$ Cf. Canellas, Exempla, lám. XXXIX.

${ }^{48} \mathrm{Cf}$. Mundó, El pacte de Cazola, lám. 8.

49 Aparece este tipo en morttem (p.c., columna 1, línea 25), mors (p.c., columna 1, línea 25), mortuis (p.c, columna 2, línea 7) mediator (p.c. columna 2, línea 9). Esta letra también se puede localizar en otros manuscritos coetáneos del siglo XII.

${ }^{50}$ Cf. CENCETI, G., Lineamenti, p. 53.

si Cf. MEYER. W.: «Die Buchstaben - Verbindungen der sogenannten gothischen Schrift», en Abhandlungen der Akademie der Wissenschaften in Göttingen. Philologisch-Historische Klasse. Neue Folge, Band, 1 (1987), 1-124. 
las cuales sólo aparecen en el presente manuscrito, y coincidiendo con el mismo fenómeno que describía Bischoff ${ }^{52}$ para los manuscritos normandos del siglo XII, los siguientes:

[a] La fusión de dos $P$, que podemos observar en Heppetic (p.c., col. 1 , 1. 4); apparuit (p.p., col. 1, 1. 12); apparebit (p.p., col. 1, I. 17) y pro peccato (p.p., col. 2, 1.2 y 8 ).

También señalaba el mismo autor que las escrituras de este período elles ne possédent pas encore, ou seulement dans des cas exceptionels, les "ligatures de courbes", les Bogenverbindungen... ${ }^{53}$.

Sólo se observa en el presente manuscrito un pretendido acercamiento de los trazos curvos, y especialmente entre la $d$ uncial y e (quidem, p.c., col. 1, 1. 16; Foderunt, p.c, col. 1, 1. 23; intercedente y mediator, p.c., col. 2 , 1. 10; hisdem, p.p., col. 1, 1. 22). El resto ni siquiera presenta este acercamiento.

Deberíamos señalar, además, que existen otros dos nexos en el texto que comentamos:

[b] La extraña fusión localizada entre la $M$ y la $E$. En este caso, el tramo final de la primera sirve de base para la formación de la segunda (sermonem, p.c., col. 1, I. 9).

[c] La fusión ac en apacasti (p.p., col. 2, 1.1).

[d] El enclave formado por la Q y la D en la abreviatura quod (p.c., col. 1, 1. 15).

\section{LIGADURAS}

Tomando como base la definición de G. Cencetti, son collegamenti spontanei e naturali di due o piu lettere fra loro, determinati da motivi grafici e per tanto frequentissimi nelle scritture corsive ${ }^{54}$. Las podemos reducir a dos grupos:

[a] las clásicas $C T$ y $S T$.

$52 \mathrm{Cf}$. Bischoғ, B. «L a nomenclature des écritures livresques du $\mathrm{IX}^{\mathrm{c}}$ au $\mathrm{XII}^{\mathrm{e}}$ sièclen, publicado en *Nomenclature des écritures livresques du $\mathrm{IX}^{\mathrm{e}}$ au $\mathrm{XVI}^{e}$ siècles\%, premier colloque International de Paléographie Latine, Paris, 28-30, avril, 1953, Paris 1954, p.11.

${ }^{3}$ Cf. Ibíd.

${ }^{54}$ Cf. Cencetr, G. Lineamenti, p. 53. 
[b] las ocasionadas por la fusión o encuentro de la parte final de un trazo y el punto de ataque del primero de la letra siguiente, que se producen con frecuencia.

Ciñéndonos, por consiguiente, a las del primer grupo, observamos que si bien la ligadura $S T$ se mantiene igual que en períodos escriturarios anteriores, diferenciándose únicamente por el truncamiento recibido (in isto, p.c., col. 1, 1. 15; assistens, p.c., col. 1, 1. 28; testamenti, p.c. col. 2, 1. 9; testamentum, testatoris, p.c., col. 2, 1. 15-16; destitucionem, ostiam, ststutum, p.c. col. 1, 1. 11-13; hostiis, potest, p.p. col. 1, 1. 22 y 24; apacasti, p.p., col. 1, 1.1). Por el contrario, en la de CT, del trazo alto de unión de ambas letras, sólo queda un recuerdo, resultando artificioso y meramente estético (factum, p.c., col. 1, 1. 30; expectantibus, p.p., col. 1, 1. 17; perfectos, p.p., col. 1, 1. 24). Esta simplificación de la ligadura $C T$ también la hemos localizado en los manuscritos y documentos con los que hemos venido comparando el presente. Así, se encuentra en:

[a.1] En 1151, en la palabra facta del escatocolo del documento de Ramón Berenguer $\mathrm{IV}^{55}$.

[a.2] En 1164, en predictam (1.7) y predictum $(1.11)^{56}$.

[a.3] En 1166, en facta $(l, 10)^{57}$.

[a.4] En 1171, se mantiene, al contrario, la forma completa de la ligadura ${ }^{58}$.

[a.5] En 1179, vuelve a presentamos la misma forma artificiosa que en ejemplos anteriores en cunctis ( $(1.1)$, spectare (1.2), dictum y actum $(I .6)^{39}$.

[a.6] En 1188 ha desaparecido la unión, y sólo queda una pequeña inclinación descendente en el punto álgido de la $t$ (coniunctionis, 1.1 )

[a.7] En 1192 en las muestras que Mundó reproducía del Liber Feudorum Maior también aparecía un trazo artificioso en la $T$, que no llegaba a unirse con $C$ (facta, lám. 8, col. 1, 1. 14; dicti, lám. 8, col. 2, 1. 3; octuber, lám. 10, col. 2, 1. 14; Factum, lám. 14, col. 1, 1. 13) ${ }^{61}$. Mundó lo describía de la siguiente forma: $C T$ digraf en el que el nexe originari entre les dues lletres s'ha convertit totalment artificiós, desenganxat de la $c i$ sovint amb un atac marcat ${ }^{62}$.

[a.8] Y, finalmente, en el manuscrito De civitate Dei, n. 20, del Archivo de la Catedral de Tortosa nos presenta dos posibilidades: una en la que la ligadura $C T$ aparece de forma completa (infructuosum, col. $2,1,11$ ), y otra en la que aparece

55 Cf, Canellas, A. (ed.), Colección diplomática, facs. 3.

56 Mateu Ibars, J. y M.D., Coleciánea, lám. 59.

57 Íd. lám. 60.

${ }^{58} \mathrm{Cf}$. CANEu, As, A., Exempla, pars altera, lám. XXXIX, Vid. factus (línea 9), cunctaque (línea 29), Recte (columna 2, línea 8) y cunctis (columna 2, línea 28).

${ }^{59} \mathrm{Cf}$. Mateu IBars, J y M.D., Colectánea, lám. 63 b.

60 Cr. KIRCHNER, Scriptura Gothica Libraria, Tab. 6.

${ }^{61}$ Cf. Mundó, A.M., aEl Pacte de Cazolas. Vid. selección de láminas.

${ }^{62} \mathrm{Cf}$. Mundó, A. M., El Pacte de Cazola, p. 125. 
de forma artificiosa el trazo de la T y por tanto, incompleta (recte, expectantibus, col. $1,1.16$ y $26 ;$ dicta, col. $2,1.6)^{63}$.

\section{ABREVIATURAS}

A continuación presentamos una selección de las abreviaturas que aparecen en el membrum disiectum, clasificadas según el esquema de estudio propuesto por A. Capelli en su Dizionario ${ }^{64}$. En algunos casos la abreviatura irá acompañada de los años en que hemos localizado su utilización, bien sea a través de las colecciones braquigráficas o por la aparición de las mismas en determinados manuscritos recogidos por las colecciones de facsímiles que venimos utilizando y que han servido para datar otras particularidades gráficas presentes en el texto de Lavaix.

\section{Truncamiento normal}

$\mathrm{h}^{\prime}($ con punto sobrescrito $)=$ hoc $(\mathrm{col}, 1,1.8)^{65}$

$\mathrm{N}($ con línea sobrescrita $)=$ non $(\mathrm{col} .1,1.12)^{66}$

$\mathrm{A} \bar{n}($ con línea sobrescrita sobre la $n)=$ ante $(\mathrm{col} .1,1.23)^{67}$

Dixer $($ con línea sobre $\mathrm{r})=$ dixerunt $(\operatorname{col} .1,1.7)^{68}$.

Etiä $\left(\right.$ con línea sobre la a) $=\operatorname{etiam}(\text { col. } 2,1,27)^{69}$

63 Cf. Mateu Ibars, J. y M.D., Colectáned, lám. 74. XIIX.

64 Cf. CAppeI.I, A. Lexicon Abbreviaturanum. Dizionario de abbreviature, p. XII .

No hemos utilizado los diccionarios españoles de abreviaturas porque carecen de indicaciones cronológicas (RIEsco TERRERo, A., Diecionario de abreviaturas hispanas de los siglos Xirr al XVIu. 1983. SEVILLANO COLOM, F., Ensayo de un elenco de abreviaturas paleogrúficas medievales, Valencia 1943.

${ }^{65}$ Cf. Mateu lBars, J. y M.D., Colectánea, lám. 72, línea 12. Canfil as, A., Colección, lám. 3. Suscripción de Pere Capella; Matzu liars, Colectánea, lám. 63 b. línea 7 entre otras; Mundo, A.M., El Pacte de Cazola, lám. 3, suscripción de Ramón de Sitges; Mateu IBars, Colectánea, lám. 64, en la suseripción notarial; id., lám. 73, linea 11, perviviendo inclaso en 1240, véase Caneluas, A. Exempla, Pars Aliera, lám. 46, línea 18. CAPPEllit data esta abreviatura en la mitad del siglo XII. Cf. Dizionario, p. 156.

${ }^{66}$ CAPpeL. L la data en el siglo XII. CF. Dizionario, p. 229.

${ }^{67}$ De mediados del siglo XI, véase CAppziLI, Dizionario, p. 16.

68 CAPpelli la data en el siglo xay. Cf. Dizionario, p.103, al igual que la de dix por dixit. Sin embargo hemos encontrado en 1193 dos abreviaturas en los verbos video y audio, en los que la desinencia de la tercera persona del plural (-unt) se omitia con el signo general de abreviación, cf. MATEU IBARS, Colectánea, lám. 67, viderunt et audierunt, líncas 17-18. La misma abreviatura puede observarse en Firmaverum, Foderunt, inciderwit, de nuestra lista.

69 CAppelu incluye en el Dizionario una abreviatura igual que ésta pero en escritura visigótica y datada $X I I$ p., p. 124. 


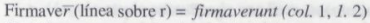

Un $($ línea sobre $\mathrm{n})=$ Unde $(\mathrm{col}$. $1, I .5)$.

\section{Truncamiento mixto}

At $($ línea sobre $\mathrm{t})=$ autem $(\mathrm{col} .1,1.26)^{70}$

Cfirmac (líneas sobre $f$ y $c)=$ confirmacione $(\mathrm{col} .1,1.4)^{71}$

Hō i e (línea sobre vocales) $\approx$ hominem $(\mathrm{col} .1, I .11)$

Oe $($ línea sobre vocales $)=$ omnem $($ col. 2, 1.24).

\section{Contracciones puras}

Ipe $($ línea sobre e $)=$ ipse (p.c., col. 1, 1.25)

$\mathrm{Qd}=$ quod (p.c., col. $1,1.15)^{72}$

$\mathrm{Tc}=$ tunc $($ p.p., col. $2,1.3$ y 10).

\section{Contracciones impuras}

Hō i $\mathrm{e}=$ homine (p.c., col. $1,1.15)$

pc $\bar{c} \bar{a}=$ peccata (p.p., col. $1,1.16)$

pc $\overline{\mathrm{r}} \hat{\mathrm{i}}=$ pecari $($ p.p., col. $1,1,11)$.

70 Esta abreviatura era localizada por CAPPEL. I, en los siglos XIV XV, Dizionario, p. 26.

n La presente abreviatura tal y como aparece en este manuscrito no está recogida por CAppeu, aunque existen otras en las que la sílaba con se abrevia solamente con la $C$ y el signo general, Dizionario, p. 51 (Cf. confinio; Cfcis: confectis; Cfcm: confectum. La primera datada a finales del siglo xul y las otras dos en el siglo XV). Esta costumbre de utilizar el signo de cum, con, también la hemos localizado en otros manuscritos, concretamente en una escritura carolina, datada por las profesoras MATEU IBARs en el siglo XI-XI, en la palabra consuetudo. línea 18.

72 La abreviatura de quod, en la cual forman un enclave la $Q$ y la $D$ minúsculas, la hemos localizado también en los siguientes manuscritos:

- En 1169 en un documento de la zona de Tudela publicado por Matey Irars, Colectánea, lám. 61, línea 19.

- En 1171 en la lámina XXXIX publicada por CANEu as, Exempla, Pars altera. línea 27.

- También lo encontramos en el facsímil XXXVII de los Exempla de Caneulas del siglo XII, en la línea 19.

- Caprel.u en el Dizionario, p. 306, data esta abreviatura en los siglos XII-XII. 
7 (siete) $=e t^{73}$

$2($ signo semejante a un dos $)=-$ ur $\left(\right.$ Frenare $t^{2}=$ frenaretur, p.c, col $\left.1, I .1\right)$

?' (coma o interrogante descendente con diferentes valores) $=$ us, - ost

-Acri' (coma sobrescrita) $=$ acrius (p.c., col. 1, 1. 15).

-corp'(íd.) $=$ corpus (p.p., col. 2, 1.1)

-Holoca' (id.) = Holocaus (p.p., col. 2, 1.1 y 7)

$-p^{\prime}=$ post $(\text { p.p., col. } 1,1.14)^{1 / 4}$

$R($ dos atravesado por línea inclinada $)=-$ rum $(\mathrm{cf}$. bonorum, p.c., col. 1 , 1. 29); futurorum (p.p., col. 1, 1. 20); taurorum (p.c., col. 2, 1. 2) ${ }^{75}$.

\section{Abreviaturas por signos abreviativos con significado relativo}

p $(\mathrm{p}$ cortada $)=\operatorname{per}($ p.c., col $.1,1.29)$

$\mathrm{p}(\mathrm{p}$ con doble ojuelo $)=$ pro $($ p.p., col. $1,1,4)$

; (punto y coma) =-ed o-ue (sed, p.c., col. 1, 1. 2, 20, 25, 32); neque (p.c., col. 1, 1.31).

" $($ doble coma $)=-$ uia $(\text { quia, p.c., col. } 1,1.26)^{76}$

- (línea sobre $q)=-u e(q u e, p . c ., c o l .2, I .11)$

a $($ cursiva sobre la $q)=-u a(q u a n t o, p . c .$, col. $2,1.4$; nunquam, p.p., col. $1,1.23)^{77}$

73 También hemos localizado la utilización de la ligadura ET (p.c. columna 1, línea 16; columna 2, línea 9; p. columna 1, línea 12) en manuseritos del siglo xn. Mundó la describía del siguiente modo: et en nexe clàssic molt inclinat a dreta $i$ amb el darrer traf que talla el precedent. Cf. Mundó, El pacte de Cazola, p. 125, véase también los ejemplos incluidos en $\mathrm{D}_{2}$, D3 y L.F.M. de la lámina número $17 \mathrm{en} \mathrm{el} \mathrm{mismo} \mathrm{artículo.} \mathrm{Formas} \mathrm{similares} \mathrm{a} \mathrm{las} \mathrm{que}$ presenta esta ligadura en nuestro manuscrito las bemos encontrado también en MATEU IBARS, J. y M.D., Colectánea, lám. 57 (línea 4); lám. 60 (línea 3), lám. 61 (línea 17), 63 a (línea 4, hay 3 ejemplos); lám. 63 b (línea 1 ).

${ }_{74}$ Localizada esta abreviatura en 1181 (cf. Mateu IBARS, J. y M.D., Colectánea, lám. 64. línea 9) y en un manuscrito datado en el mismo siglo (cf. MATEU IBARS, J. y M.D., Colectánea, lám. 74, línea 20 de la columna 1).

${ }^{75}$ Es interesante comparar la forma adoptada por este signo abreviativo con los otros que aparecen a lo largo del texto. En el de Bonorum de la pars pili (columna 1) la estructura del mismo se asemeja más a los precedentes carolinos, observando únicamente el goticismo en las curvas, comparable al utilizado en la lámina 58 de la Colectánea de 1164 (sanctorum, línea 19),

${ }^{76}$ Una forma similar aparece en el facsímil XXXIX de 1171 de la colección Exempla de A. Caneri as (línea 3, columna 1). CAppes s en su Dizionario, p. 302, databa un grafismo parecido con valor de quia para el siglo $\mathrm{xIII}$.

77 La misma estructura que la adoptada por la abreviatura quam la encontramos en los siguientes manuscritos: de 1179, MATBu IBARS, Colectánea, lám. 63, línea 3; de 1181, id. lám. 64, línea 4; id, lám. 73, del siglo XII, columna 2, línea 13, y también en el Liber Feudorum Maior, cf. Mundó, A., El pacte de Cazola, lám. 10, línea 8 (Nunquam): lám. 11, línea 17 (tanquam). 
$>$ (ángulo agudo atravesando una $\mathrm{S})=-$ er $($ sermonem, p.c., col. 1, 1.2)

$>($ atravesando $\mathrm{L})=-$ ul $($ Immaculatum, p.c., col. 2,1 . 6)

\section{Abreviaturas por letras sobrescritas}

a, e, i, o (sobre q) =-ua, -ue, -ui, -uo (aqua, p.c., col. 2. 1. 23; quanto, p.c. col 2, 1. $4^{78}$; (que, p.c., col. 1, 1. 2); (alioquin, p.c., col. 2, 1. 18; iniquitas, p.c., col. 1, 1. 26); (quod, p.p., col. 1, 1.26).

$\mathrm{e}($ sobre $\mathrm{C})=-$ re $($ creacionis, p.c., col. $1,1.31)$.

$\mathrm{i}($ sobre $\mathrm{m})=\operatorname{mihi}($ p.p., col. $2,1,1)$

$\mathrm{i}($ sobre $\mathrm{n})=$ nisi $($ p.c., col. $1,1,8)$

$\mathrm{i}($ sobre $\mathrm{p})=-$ ri (priori, p.c., col. $2,1.12)$

$\mathrm{i}($ sobre $\mathrm{G})=-$ ui $($ sanguine, p.c., col. $2,1.20)$

$\mathrm{i}($ sobre $\mathrm{S})=\operatorname{sibi}($ p.c., col. $1,1.2)$

$\mathrm{i}($ sobre $\mathrm{T})=$ tibi $($ p.p., col. $2,1.2)$

$\mathrm{i}($ sobre $\mathrm{X})=\operatorname{Christi}($ p.c., col. $2,1.15)$

o $($ sobre $g)=\operatorname{ergo}(\text { p.c., col. } 1,1.15)^{79}$

$o($ sobre $\mathrm{t})=-$ ro $($ Introitu, p.c., col. $1,1.33)$

$\mathrm{o}($ sobre $\mathrm{Q}$ y sobre $\mathrm{m})=$ quomodo $(\text { p.c. col. } 1,1.5)^{80}$.

\section{CONCLUSIÓN}

El estudio comparado que hemos efectuado de este «Membrum disiectum" con otros manuscritos, documentales y librarios, procedentes de la zona del Pirineo ${ }^{81}$, nos ha dado los siguientes resultados:

78 Véase nota anterior.

79 La abreviatura de ergo por letra final sobrepuesta la databa CAPpelu en su Dizionario a fines del siglo XIV (p. 148). Sin embargo la misma abreviatura la hemos localizado en 1171 en CANel l.as, Exempla, Pars altera, lám. XXXIX, línea 10 de la columna 1.

${ }^{80} \mathrm{La}$ abreviatura de quomodo, con dos $O$ sobrescritas a $Q$ y $M$ era datada por CAppel. $\mathrm{con}$ el Dizionario a principios del siglo XIV (p. 310). En la ćpoca a la que pertenece el manuscrito que estamos estudiando ya existía csta forma de abreviar la palabra. Algunos ejemplos de su uso en la Península Ibérica nos los muestran los siguientes manuscritos: en 1151 el documento de Ramón Berenguer IV que ya hemos comentado, Cf. CaNellas (ed,), Colección Diplomáti$c a$, facsímil 3 (línea 1.1) y un manuscrito cronológicamente anterior, reproducido por la lámina 72 de la Colectánea (línea 17), en escritura carolina,

$81 \mathrm{Cf}$. el mapa adjunto donde se localizan los topónimos y años de datación de los documentos que hemos utilizado para efoctuar la datación según las colecciones de facsímiles que hemos usado. Agradezco a Adolfo Calvo Cases las facilidades prestadas para la elaboración de este mapa. 
a) Que el límite a quo puede venir representado por el documento de Ramón Berenguer IV del año 1151, expedido en Zaragoza, y el límite final, ad quem, por el Liber Feudorum Maior, que según Mundó fou acabat i presentat al rei en $1192^{82}$.

b) Que el «membrum disiectum» procedente de Lavaix es anterior a la fecha de finalización del cartulario real, porque éste - a tenor de las reproducciones que nos muestra Mundó- presenta una estructura gráfica totalmente quebrada, pudiendo representar fácilmente la base que posteriormente dará lugar a las Textuales Caligráficas de los primeros años del siglo $\mathrm{XII}^{83}$.

c) Que si atendemos a sus alzados, y especialmente en sus puntos de ataque, podríamos localizarlo hasta el año $1193^{84}$.

d) Que, por el contrario, el tratamiento recibido por el signo general de abreviación sobre la $L$, la ligadura de $E T$, y por la forma redondeada adoptada por uno de los signos abreviativos de -rum — todos los cuales reflejan ciertas reminiscencias carolinas diferentes del tratamiento general de todos los grafismos del texto- nos llevaría a situarlo entre los primeros años incluidos en los límites, inicial y final, o incluso en alguna fecha anterior.

e) Que la no estricta observancia de las reglas de W. Meyer, como detectaba Bischoff, también lo situaba en esta época.

f) Respecto a la zona geográfica, sólo sabíamos al comienzo del trabajo que provenía del monasterio de Lavaix ${ }^{85}$, aunque desconocemos si había sido escrito en esta zona o se había conservado allí, hecho que demostraba la aparición de este fragmento en un paquete de documentación procedente del mencionado cenobio. Sus formas gráficas, similares a la producción gráfica pirenaica, nos llevaban a situarlo dentro de la actividad caligráfica de esta zona y concretamente dentro de los límites marcados por el Ebro y el Segre. Sólo escapan a estos territorios un manuscrito de Veruela de 1171 y el documento de Tarazona de 1193 por la parte occidental, aunque gráficamente son muy similares a los incluidos en los límites territoriales. El desplazamiento representado por Barcelona, por la escritura de Ramón de Sitges y el Liber Feudorum Maior, son justificables por la presencia de estructuras gráficas totalmente quebradas, ante-

${ }^{82}$ Cf. Mundó, A. El pacte de Cazola, p. 126.

83 Cf. Canellas, A., Exempla Scripturarum. Pars Altera, lám. XLVI, de 1240, que reproduce un folio del Fuero de Teruel.

${ }_{4}^{4}$ Cf. Mateu IaArs, J y M.D., Colectínea, lám. 67.

85 Cf. LINAGE CONDE, A, Los origenes del monacato benedictino en la Península Ibérica, León 1973, vol. II, mapa II, vol. III, p. 225-226. 
riores al documento de Tarazona de 1193. ¿Pudo ser porque los manuscritos de Barcelona proceden del mundo urbano frente al mundo rural que envuelve a Tarazona? ¿Podrían explicarse aduciendo que el mundo urbano es mejor receptor de nuevas corrientes gráficas o que su evolución es más rápida?

g) Finalmente, el hecho de que el manuscrito se localice en esta zona cuestiona la afirmación realizada por $\mathrm{F}$. Balaguer ${ }^{86}$, quien opinaba que la escritura carolina fue introducida en Aragón como consecuencia de la relación mantenida entre monasterios aragoneses y franceses durante el reinado de Ramón Berenguer IV. ¿No se trataría de formas góticas? Otra pregunta obligada sería la relativa a la posible función de intermediaria desempeñada por esta zona en la introducción de este tipo gráfico en Aragón proveniente de los Condados catalanes, al igual que sucedió con determinados elementos diplomáticos, como han demostrado Trenchs y Arago ${ }^{87}$.

Una vez finalizada la investigación, y volviendo al principio del trabajo, creemos que persiste el problema de las dataciones de manuscritos. Resulta muy difícil reconocer únicamente por elementos gráficos la fecha exacta de la confección o copia de un determinado texto escrito.

\section{APÉNDICE}

$\mathrm{El}$ «membrum disiectum» que hemos intentado datar con el presente trabajo pertenece a la colección privada de J. Trenchs, a quien agradecemos la facilidad concedida para su estudio.

Se trata de un pergamino, bastante deteriorado en el tercio inferior y escrito por ambas caras; presenta iniciales coloreadas en rojo, y con la eforatura di riga" al margen derecho del recto; el vuelto fue utilizado en fecha posterior para realizar unas operaciones matemáticas en caracteres arábigos.

Contiene textos del Nuevo Testamento, concretamente de S. Mateo y de la Epístola a los Hebreos.

86 Cf. BALAGUER, F., «Una nota sobre la introducción de la letra carolina en la Cancillerfa aragonesas, Cuadernos de Historia, Jerónimo Zurita, 3 (1952), p. 155-161.

87 Cf. ARAGo, A.M., y TRENCHS, J., «Las escribanías catalano-aragonesas de Ramón Berenguer IV a la minoría de Jaime Is, Revista de Archivos, Bibliotecas y Museos, LXXX (1977), p. 421-442, y especialmente la 425. 


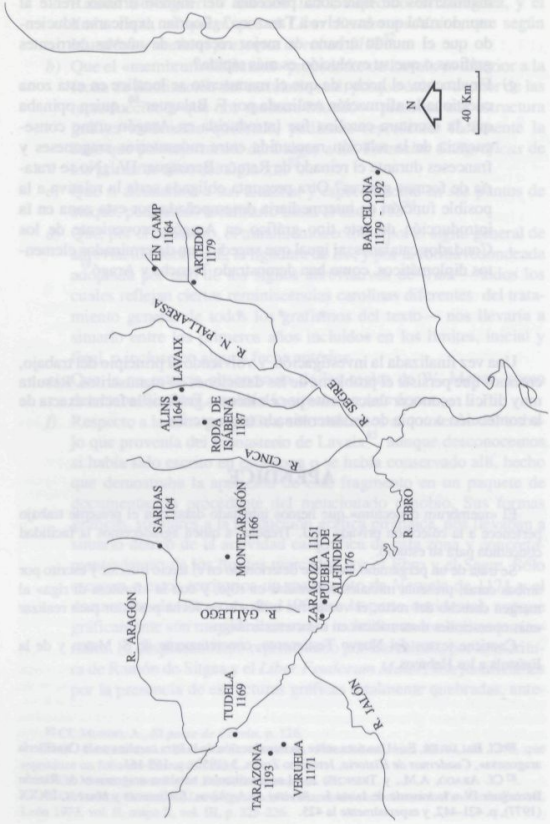

